\title{
Spectral gradient methods for linearly constrained optimization
}

\author{
J. M. Martínez * \\ E. A. Pilotta ${ }^{\dagger}$ \\ M. Raydan $\ddagger$
}

May 27, 2003

\begin{abstract}
Linearly constrained optimization problems with simple bounds are considered in the present work. First, a preconditioned spectral gradient method is defined for the case in which no simple bounds are present. This algorithm can be viewed as a quasiNewton method in which the approximate Hessians satisfy a weak secant equation. The spectral choice of steplength is embedded into the Hessian approximation, and the whole process is combined with a nonmonotone line search strategy. The simple bounds are then taken into account by placing them in an exponential penalty term that modifies the objective function. The exponential penalty scheme defines the outer iterations of the process. Each outer iteration involves the application of the previously defined preconditioned spectral gradient method for linear equality constrained problems. Therefore, an equality constrained convex quadratic programming problem needs to be solved at every inner iteration. The associated extended KKT matrix remains constant unless the process is reinitiated. In ordinary inner iterations, only the right hand side of the KKT system changes. Therefore, suitable sparse factorization techniques can be effectively applied and exploited. Encouraging numerical experiments are presented.
\end{abstract}

Key words: Linearly constrained optimization, quasi-Newton methods, exponential penalty methods, spectral gradient method, nonmonotone line search.

*Department of Applied Mathematics, IMECC-UNICAMP, University of Campinas, CP 6065, 13081970 Campinas SP, Brazil (martinez@ime.unicamp.br). This author was supported by FAPESP (Grant 2001-04597-4), FINEP and FAEP-UNICAMP.

${ }^{\dagger}$ Department of Applied Mathematics, IMECC-UNICAMP, University of Campinas, CP 6065, 13081970 Campinas SP, Brazil (pilotta@ime.unicamp.br). This author was supported by FAPESP (Grant 903724-6), FINEP and FAEP-UNICAMP.

${ }^{\ddagger}$ Departamento de Computación, Facultad de Ciencias, Universidad Central de Venezuela, Ap. 47002, Caracas 1041-A, Venezuela (mraydan@reacciun.ve). Work sponsored by FAPESP, FAEP-UNICAMP and the Scientific Computing Center at UCV. 


\section{Introduction}

Spectral gradient methods have proved to be of great value in unconstrained optimization problems. They were introduced by Barzilai and Borwein [1], and later analyzed by Raydan [21]. They have been applied to find local minimizers of large scale problems $[4,5,22]$, and also to explore faces of large dimensions in box-constrained optimization (see [3] and [12]). More recently, spectral gradient methods were extended to minimize general smooth functions on convex sets. See [6]. In this case, the spectral choice of steplength was combined with the projected gradient method to obtain a robust and effective low cost computational scheme.

In this work, we present a spectral gradient method for the linearly constrained optimization problem

Minimize $f(x)$ subject to $A x=b, x \geq 0$,

where $f: \mathbb{R}^{n} \rightarrow \mathbb{R}$ is a smooth function. We consider, first, the case in which the bounds $x \geq 0$ are not present. For this case, we present a quasi-Newton method in which the secant approximation satisfies a weak secant equation. Our method can also be viewed as a preconditioned spectral gradient method (see $[17,19]$ ), in which the secant update plays the role of a preconditioner matrix that can be reinitialized whenever some indicator of performance reveals that this is convenient. The spectral choice of steplength is embedded into the secant matrix via a simple Rayleigh quotient scalar multiplication, and the whole process is combined with a nonmonotone line search strategy. The simple bounds are then taken into account by means of an exponential penalty term that modifies the objective function. Each modification of the penalty term defines a different outer iteration. At each outer iteration we apply the preconditioned spectral gradient method for linear equality constrained problems defined before.

Concerning the implementation, a basis for the null space of $A$ is not required at all. To obtain the search direction we need to solve a convex quadratic programming problem at every inner iteration. As a consequence of the spectral choice of steplength, the associated extended KKT matrix remains constant unless the process is reinitialized, and only the right hand side of the KKT system changes. Therefore, suitable sparse factorization techniques can be effectively exploited.

This work is organized as follows. In section 2 we present the spectral gradient method for linear equality constrained problems. We describe the calculation of the search direction, the nonmonotone line search and the convergence properties of the method. In section 3, we present an exponential penalty approach to solve the linearly constrained optimization problem with simple bounds. In section 4 we give a global convergence result for the proposed outer scheme. In section 5 , we describe implementation features. In section 6 , we present computational results. Finally, in section 7 , we state some conclusions. 


\section{Linearly constrained preconditioned spectral gradient with- out bounds}

Let us consider the problem

$$
\text { Minimize } F(x) \text { subject to } A x=b,
$$

where $F: \mathbb{R}^{n} \rightarrow \mathbb{R}, A \in \mathbb{R}^{m \times n}, F \in C^{1}\left(\mathbb{R}^{n}\right)$, and $b$ lies in the range of $A$. We denote $g(x)=\nabla F(x)$ for all $x \in \mathbb{R}^{n}$.

In this section we define a quasi-Newton algorithm for solving (1). Assume that $0<\alpha_{\min }<\alpha_{\max }<\infty$. Given $x_{k} \in \mathbb{R}^{n}$ such that $A x_{k}=b$ and a positive definite matrix $B_{k}$ (this can be relaxed to $Z^{T} B_{k} Z>0$, where the columns of $Z$ are a basis of the null space of $A$ ), the steps for obtaining $x_{k+1}$ are given by the following algorithm.

Algorithm 1. Preconditioned spectral gradient with linear equality constraints

Step 1: Obtain $d_{k} \in \mathbb{R}^{n}$ the unique solution of

$$
\text { Minimize } \frac{1}{2} d^{T} B_{k} d+g\left(x_{k}\right)^{T} d \text { subject to } A d=0 .
$$

If $d_{k}=0$ terminate. $\left(x_{k}\right.$ is a stationary point of problem (1))

Step 2: Compute, using a procedure that will be specified later (in Algorithm 2), $\lambda_{k}>0$ (the steplength). Define

$$
\begin{gathered}
x_{k+1}=x_{k}+\lambda_{k} d_{k}, \\
s_{k}=x_{k+1}-x_{k}, \\
y_{k}=g\left(x_{k+1}\right)-g\left(x_{k}\right), \\
B_{k+1}=\alpha_{k} B_{0},
\end{gathered}
$$

where if $s_{k}^{T} y_{k}>0$ then

$$
\alpha_{k}=\max \left(\alpha_{\min }, \min \left(\alpha_{\max }, \frac{s_{k}^{T} y_{k}}{s_{k}^{T} B_{0} s_{k}}\right)\right)
$$

else $\alpha_{k}=\alpha_{\min }$.

Return to Step 1.

The solution of (2) is given by

$$
\left[\begin{array}{cc}
B_{k} & A^{T} \\
A & 0
\end{array}\right]\left[\begin{array}{l}
d_{k} \\
\psi_{k}
\end{array}\right]=\left[\begin{array}{c}
-g_{k} \\
0
\end{array}\right]
$$

where $g_{k}=g\left(x_{k}\right)$. The matrix of the linear system is referred as the KKT-matrix. Note that in the particular case in which $B_{k}=I$, the solution $d_{k}$ is the orthogonal projection of $-g\left(x_{k}\right)$ on the null-space of $A$. 


\section{Remarks}

1. With the appropriate changes on the bounds for $\alpha_{k}$ we can define $B_{k+1}=\alpha_{k} B_{k}$ using $\frac{s_{k}^{T} y_{k}}{s_{k}^{T} B_{k} s_{k}}$ instead of $\frac{s_{k}^{T} y_{k}}{s_{k}^{T} B_{0} s_{k}}$. In this way it is easier to visualize the algorithm as a quasi-Newton method in which, when the bounds for $\alpha_{k}$ are not violated, the matrix $B_{k+1}$ satisfies the "weak secant equation"

$$
s_{k}^{T} B s_{k}=s_{k}^{T} y_{k} .
$$

The geometrical meaning of $(5)$ is that the directional derivative of the quadratic model of $F$ coincides with the directional derivative of $F$ at the previous point. Obviously, the gradient of the quadratic model coincides with the gradient of $F$ at the current point. With our spectral choice, the directional derivatives of the quadratic model on directions that are $B_{k}$-orthogonal to the increment coincide with the directional derivative of $F$ at the current point.

2. If no constraints are present at all and $B_{0}=I$ this algorithm is, essentially, the one defined by Raydan in [22]. The present algorithm generalizes also in many senses the preconditioned spectral gradient method introduced in [17], where $B_{0}$ plays the role of the preconditioner matrix.

3. The quantity $\frac{s_{k}^{T} y_{k}}{s_{k}^{T} B_{0} s_{k}}$ is frequently referred as a Rayleigh quotient. In fact, if $F \in$ $C^{2}\left(\mathbb{R}^{n}\right)$, we have:

$$
y_{k}=\left[\int_{0}^{1} \nabla^{2} F\left(x_{k}+t s_{k}\right) d t\right] s_{k} .
$$

So, defining $w_{k}=B_{0}^{\frac{1}{2}} s_{k}$ and

$$
A_{k}=B_{0}^{-\frac{1}{2}}\left[\int_{0}^{1} \nabla^{2} F\left(x_{k}+t s_{k}\right) d t\right] B_{0}^{-\frac{1}{2}},
$$

we obtain:

$$
\frac{s_{k}^{T} y_{k}}{s_{k}^{T} B_{0} s_{k}}=\frac{w_{k}^{T} A_{k} w^{k}}{w_{k}^{T} w_{k}}
$$

Therefore, $\frac{s_{k}^{T} y_{k}}{s_{k}^{T} B_{0} s_{k}}$ is a Rayleigh quotient of $A_{k}$ in the classical sense.

In Algorithm 2 we describe the line-search procedure, that is to say, the way in which we choose $\lambda_{k}$ at each iteration. We adopted a nonmonotone line-search strategy. This means that, following $[6,15,22]$, we do not impose decrease of the objective function at every iteration. Instead, we choose a positive integer $M$ at the beginning of the process and we accept a trial point when a sufficient decrease is obtained in relation to the maximum functional value among the $M+1$ last iterations. 
Algorithm 2. Nonmonotone line-search procedure

We assume that $\gamma \in(0,1)$ is given independently of the iteration number $k$ and that $d_{k}$ has been computed using (2).

Step 1 Set $\lambda \leftarrow 1$.

Step 2 Set $x_{+}=x_{k}+\lambda d_{k}$.

Step 3 If

$$
F\left(x_{+}\right) \leq \max _{\left.0 \leq j \leq \min _{\{k, M}\right\}} F\left(x_{k-j}\right)+\gamma \lambda\left\langle d_{k}, g\left(x_{k}\right)\right\rangle,
$$

then define $\lambda_{k}=\lambda$ and finish the line-search.

If (6) does not hold, define

$$
\lambda_{\text {new }} \in[0.1 \lambda, 0.9 \lambda],
$$

set $\lambda \leftarrow \lambda_{\text {new }}$ and go to Step 2 .

Algorithm 1 admits many possible implementations according to the choice of the matrix $B_{0}$. The "pure" spectral gradient method with linear constraints corresponds to $B_{0}=I$. Our approach here, similarly to the one of [17], is to consider that Algorithm 1 could be re-initiated with a different initial matrix $B_{0}$ whenever some indicator of performance reveals that this is convenient. The whole process is described below.

\section{Algorithm 3. Spectral gradient for linear equality constraints with re-initialization}

Assume that the positive definite matrix $B_{0}$ is given, as well as the positive integer $M$ and the sufficient decrease parameter $\gamma \in(0,1)$.

Step 1. Set kount $\leftarrow 0, k \leftarrow k o u n t$

Step 2. Execute Algorithm 1 with the line-search procedure given by Algorithm 2.

Step 3. If Algorithm 1 terminates at Step 1, terminate the execution of Algorithm 3 too.

Step 4. Decide whether it is necessary to re-initiate Algorithm 1 (YES-NO).

Step 5. If NO, set kount $\leftarrow$ kount $+1, k \leftarrow k+1$ and go to Step 2 .

Step 6. If YES, define a new $B_{0}$, set kount $\leftarrow$ kount $+1, k \leftarrow 0$ and go to Step 2 .

The following observation plays an important role in our convergence analysis and also in our implementation. Let $\left(d_{k}, \psi_{k}\right)$ be a solution of the system (4). Then $d_{k}$ belongs to the null space of $A$, that is, for some $w_{k}, d_{k}=Z w_{k}$ where the columns of $Z$ form a basis of the null space of $A$. Therefore

$$
B_{k} Z w_{k}+A^{T} \psi_{k}=-g_{k} .
$$

So,

$$
Z^{T} B_{k} Z w_{k}+Z^{T} A^{T} \psi_{k}=-Z^{T} g_{k} .
$$

Since $A Z=0$ and $B_{k}$ is positive definite, we have

$$
w_{k}=-\left(Z^{T} B_{k} Z\right)^{-1} Z^{T} g_{k},
$$


and hence

$$
d_{k}=-Z\left(Z^{T} B_{k} Z\right)^{-1} Z^{T} g_{k} .
$$

Now, using the update for the matrix $B_{k}$ in Algorithm 1, we obtain

$$
d_{k}=-Z\left(Z^{T} B_{0} Z\right)^{-1} Z^{T}\left(\frac{g_{k}}{\alpha_{k-1}}\right) .
$$

Therefore, the search direction $d_{k}$ can be obtained using the matrix of the last re-initialization and the corresponding independent vector by means of

$$
\left[\begin{array}{cc}
B_{0} & A^{T} \\
A & 0
\end{array}\right]\left[\begin{array}{l}
d_{k} \\
\psi_{k}
\end{array}\right]=\left[\begin{array}{c}
-\frac{g_{k}}{\alpha_{k-1}} \\
0
\end{array}\right]
$$

Our next result establishes the convergence properties of Algorithm 3.

Theorem 1. Suppose that at all initializations of $B_{0}$ the eigenvalues of $Z^{T} B_{0} Z$ are clustered in a strictly positive closed and bounded interval. Then Algorithm 3 is well defined and every limit point is stationary.

Proof. By the choice of $\alpha_{k}$ in Algorithm 1, the eigenvalues of $Z^{T} B_{k} Z$ are clustered in a strictly positive closed and bounded interval. But, for all $\sigma>0$, the subproblem

$$
\text { Minimize } \frac{1}{2} d^{T} B_{k} d+g\left(x_{k}\right)^{T} d \text { s.t. } A d=0
$$

is equivalent to

$$
\text { Minimize } \frac{1}{2} d^{T}\left(B_{k}+\sigma A A^{T}\right) d+g\left(x_{k}\right)^{T} d \text { s.t. } A d=0 .
$$

So, without loss of generality, we can assume that the eigenvalues of $B_{k}$ are clustered in a strictly positive compact interval.

Therefore, Algorithm 1 is a particular case of Algorithm 2.1 of [7]. So, the thesis is true.

\section{Adding bounds on the variables}

Now, we consider the following linearly constrained optimization problem with simple bounds :

$$
\text { Minimize } f(x) \text { subject to } A x=b, x \geq 0 \text {, }
$$

where $f: \mathbb{R}^{n} \rightarrow \mathbb{R}, A \in \mathbb{R}^{m \times n}, x \in \mathbb{R}^{n}, f \in C^{1}\left(\mathbb{R}^{n}\right)$. Our arguments below can be easily extended to a problem with simple upper and lower bounds:

$$
\text { Minimize } f(x) \text { subject to } A x=b, \ell \leq x \leq u \text {, }
$$

however we will restrict ourselves to the case (11) in order to simplify the notation. 
Problem (11) can be solved by the inexact spectral projected gradient method introduced in [7]). This involves to solve quadratic linearly constrained problems with bounds approximately, using some iterative method that allows one to obtain lower bounds of the objective function at each iteration. This can be very costly in many cases. So, in this work we decided to deal with positive constraints using a penalty approach.

There are many possibilities to do this. The reputation of the logarithmic barrier function lead us to try an interior-point scheme at early stages of this work. This idea did not work well. Roughly speaking, the first trial step almost always violates the bounds $x>0$ so that, at most inner iterations, the step must be reduced. As a consequence, if there is any benefit in the spectral choice of the step, this benefit disappears when the method is associated with the logarithmic barrier function. Of course, this does not mean that the $\log$-function is not effective when it is associated to the pure Newtonian direction, as many theoretical and practical studies show.

In order to maintain the existence of second derivatives of the objective function, we decided to consider the modified exponential penalty function $[2,18,20]$ given by:

$$
\operatorname{axp}(t)= \begin{cases}e^{t} & \text { if } t \leq 0 \\ 1+t+\frac{1}{2} t^{2} & \text { if } t>0\end{cases}
$$

As a consequence, we have to solve the following minimization subproblem for each penalty parameter $\rho$ :

$$
\text { Minimize } F(x) \equiv f(x)+\frac{1}{\rho} \sum_{i=1}^{n} \mu_{i} \operatorname{axp}\left(-\rho x_{i}\right), \text { subject to } A x=b \text {. }
$$

The meaning of $\mu_{i}$ will be given later. We are only going to assume that

$$
0<\mu_{\min } \leq \mu_{i} \leq \mu_{\max }<\infty
$$

for all $i=1, \ldots, n$. The outer algorithm consists in solving a sequence of penalty problems (12). Each penalty problem is solved using Algorithm 3. Clearly, the exponential penalty approach does not require positivity of the initial point.

\section{Global convergence}

In this section, we will show that, under certain conditions, the scheme based on successive approximate minimizations of (12) really works. Similar results, for related algorithms, were given, for example, in [2] and [18]. The outer scheme proposed in the previous section is an exponential augmented Lagrangian method. At each outer iteration, the sequence of inner steps will be given by Algorithm 1. Accordingly, an inexact stopping convergence criterion will be defined, based on computable quantities.

The following assumption guarantees that, at each outer iteration, Algorithm 1 will be able to stop. 
Assumption 1. There exists $\rho_{0}>0$ such that, if $\rho \geq \rho_{0}$ and $\mu_{i} \in\left[\mu_{\min }, \mu_{\max }\right]$, $0<\mu_{\min }<\mu_{\max }<\infty$, the level sets of $F(x)$ in (12) are bounded.

Observe that Assumption 1 is stronger than saying that $f(x)$ has bounded level sets on the feasible region $A x=b, \quad x \geq 0$, since it involves the behavior of $f(x)$ on infeasible points too. If $f(x)$ goes to $-\infty$ very quickly for $x_{i} \rightarrow-\infty$, Assumption 1 might not hold. It can be argued that, in this case, it is inexpensive to modify the definition of $f(x)$ in the infeasible region, but even this operation is risky and can create undesirable local minimizers.

Now, we are able to define the outer algorithm. Each iterate of this algorithm will be $x^{k} \in \mathbb{R}^{n}$, whose existence is guaranteed by Assumption 1 .

\section{Algorithm 4. Exponential-Lagrangian scheme}

Let $\varepsilon_{k}>0$ for all $k=0,1,2, \ldots$ be such that $\lim _{k \rightarrow \infty} \varepsilon_{k}=0$. Let $\rho_{0}$ be given by Assumption 1, as well as $\mu_{\min }$ and $\mu_{\max }, 0<\mu_{\min }<\mu_{\max }<\infty$. Let $\tau \in(0,1), \eta>1$. Initialize $\sigma_{0}=0$ and $k \leftarrow 0$.

Step 1. Choose $\mu_{i}^{k} \in\left[\mu_{\min }, \mu_{\max }\right]$ for all $i=1, \ldots, n$.

Step 2. Define

$$
F_{k}(x)=f(x)+\frac{1}{\rho_{k}} \sum_{i=1}^{n} \mu_{i}^{k} \operatorname{axp}\left(-\rho_{k} x_{i}\right)
$$

Apply Algorithm 1 for

$$
\text { Minimize } F_{k}(x) \text { subject to } A x=b \text {. }
$$

Use, as stopping criterion, the test

$$
\|d\| \leq \varepsilon_{k},
$$

where $d$ is the solution of (2). (From now on, with some abuse of notation, we will denote $d_{k}=d$ in this section.) The final iterate of Algorithm 1 so far obtained will be called $x^{k}$. Step 3. Compute $\underline{\mu}^{k} \in \mathbb{R}^{n}$ by

$$
\underline{\mu}_{i}^{k}=\mu_{i}^{k} \operatorname{axp}^{\prime}\left(-\rho_{k} x_{i}^{k}\right), i=1, \ldots, n .
$$

Step 4. Define

$$
\sigma_{k+1}=\max \left\{\left|\min \left\{\underline{\mu}_{i}^{k}, x_{i}^{k}\right\}\right|, i=1, \ldots, n\right\} .
$$

Step 5. If $\sigma_{k+1} \leq \tau \sigma_{k}$ define $\rho_{k+1}=\rho_{k}$. Else, define $\rho_{k+1}=\eta \rho_{k}$.

Step 6. Set $k \leftarrow k+1$. Go to Step 1 .

The vector $\mu^{k}$ is intended to be an estimator of the Lagrange multipliers associated to the inequality constraints. For this reason, the choice $\mu^{k}=\mu^{k-1}$ could be quite natural at Step 2 , if the safeguards defined by $\mu_{\min }$ and $\mu_{\max }$ are not violated. However, this specific choice is not necessary for proving convergence. The motivation of Step 4 of the algorithm 
is that the conditions on positivity and complementarity that relate the solution and the Lagrange multipliers can be written as

$$
\min \left\{\underline{\mu}_{i}, x_{i}\right\}=0, \quad i=1, \ldots, n .
$$

Therefore, Step 4 measures the progress related to the positivity-complementarity requirement. If this progress is satisfactory, it is not necessary to increase the penalty parameter. In our practical implementations we used $\mu_{i}^{k} \equiv 1$, so that the whole practical process can be interpreted as a penalty scheme where the tests of Step 4 determine if the penalty parameter must be increased or not.

Let us define

$$
P=Z\left(Z^{T} Z\right)^{-1} Z^{T}
$$

Therefore, $P v$ is the projection of $v \in \mathbb{R}^{n}$ on the null-space of $A$. The lemma that we prove below shows that the projection of the gradient of $F_{k}$ tends to zero when one uses the stopping criterion (13). Along the rest of this paper, $\mathbb{N}=\{0,1,2, \ldots\}$.

Lemma 1. Assume that $\left\{x_{k}\right\}_{k \in \mathbb{N}}$ is generated by Algorithm 4. Then,

$$
\lim _{k \rightarrow \infty} P \nabla F_{k}\left(x^{k}\right)=0 .
$$

Proof. By (8) and (13) we have:

$$
\lim _{k \rightarrow \infty} Z\left(Z^{T} B_{k} Z\right)^{-1} Z^{T} \nabla F_{k}\left(x^{k}\right)=0 .
$$

By the linear independence of the columns of $Z$ and the assumption on the eigenvalues of $Z^{T} B_{k} Z$, this implies that

$$
\lim _{k \rightarrow \infty} Z^{T} \nabla F_{k}\left(x^{k}\right)=0 .
$$

Then,

$$
\lim _{k \rightarrow \infty} P \nabla F_{k}\left(x^{k}\right)=\lim _{k \rightarrow \infty} Z\left(Z^{T} Z\right)^{-1} Z^{T} \nabla F_{k}\left(x^{k}\right)=0 .
$$

The iterates generated by Algorithm 4 necessarily satisfy $A x^{k}=b$ for all $k$ but some of its components can be negative. Up to now, we did not assume that the feasible set given by $x \geq 0, A x=b$ is nonempty. In the next theorem we prove that, even in the empty case, limit points of the algorithm are minimizers of the sum of weighted squared infeasibilities.

Theorem 2. Assume that $\left\{x^{k}\right\}_{k \in \mathbb{N}}$ is generated by Algorithm 4 and that $x^{*}$ is a limit point. Then, there exists $\mu^{*}>0$ such that $x^{*}$ is a global solution of

$$
\text { Minimize } \sum_{i=1}^{n} \max \left\{0,-\mu_{i}^{*} x_{i}\right\}^{2} \text { s.t. } A x=b .
$$

Proof. We consider two cases: 
(a) $\left\{\rho_{k}\right\}_{k \in \mathbb{N}}$ is bounded;

(b) $\lim _{k \rightarrow \infty} \rho_{k}=\infty$.

In Case (a), we have that for all $k$ large enough $\sigma_{k} \leq \tau \sigma_{k-1}$. Therefore, $\lim _{k \rightarrow \infty} \sigma_{k}=0$. So,

$$
\min \left\{x_{i}^{k}, \underline{\mu}_{k}\right\} \rightarrow 0 \forall i=1, \ldots, n .
$$

This implies that $x^{*} \geq 0$. So, $x^{*}$ is a global minimizer of (15) for all $\mu^{*}>0$.

Now we consider Case (b). By Lemma 1,

$$
\lim _{k \rightarrow \infty} P\left[\nabla f\left(x^{k}\right)+\frac{1}{\rho_{k}} \sum_{i=1}^{n} \mu_{i}^{k} \nabla \operatorname{axp}\left(-\rho_{k} x_{i}^{k}\right)\right]=0 .
$$

So, dividing by $\rho_{k}$, we obtain:

$$
\lim _{k \rightarrow \infty} P\left[\frac{1}{\rho_{k}} \nabla f\left(x^{k}\right)+\frac{1}{\rho_{k}^{2}} \sum_{i=1}^{n} \mu_{i}^{k} \nabla \operatorname{axp}\left(-\rho_{k} x_{i}^{k}\right)\right]=0 .
$$

Let $K$ be an infinite subset of $\mathbb{N}$ such that $\lim _{k \in K} x^{k}=x^{*}$. Since $\left\{\nabla f\left(x^{k}\right)\right\}_{k \in K}$ is bounded, we have:

$$
\lim _{k \in K} P \frac{1}{\rho_{k}^{2}} \sum_{i=1}^{n} \mu_{i}^{k} \nabla \operatorname{axp}\left(-\rho_{k} x_{i}^{k}\right)=0
$$

So,

$$
\lim _{k \in K} P \frac{1}{\rho_{k}} \sum_{i=1}^{n} \mu_{i}^{k} \operatorname{axp}^{\prime}\left(-\rho_{k} x_{i}^{k}\right) \mathbf{e}_{\mathbf{i}}=\mathbf{0},
$$

where $\left\{\mathbf{e}_{\mathbf{1}}, \ldots, \mathbf{e}_{\mathbf{n}}\right\}$ is the canonical basis of $\mathbb{R}^{n}$.

Now,

$$
\operatorname{axp}^{\prime}\left(-\rho_{k} x_{i}^{k}\right)=e^{-\rho_{k} x_{i}^{k}} \leq 1 \text { if } x_{i}^{k} \geq 0,
$$

and

$$
\operatorname{axp}^{\prime}\left(-\rho_{k} x_{i}^{k}\right)=-\rho_{k} x_{i}^{k}+1 \text { if } x_{i}^{k}<0 .
$$

So, if $x_{i}^{*} \geq 0$, since $\mu_{i}^{k}$ is bounded above,

$$
\lim _{k \in K} \mu_{i}^{k} \frac{1}{\rho_{k}} \operatorname{axp}^{\prime}\left(-\rho_{k} x_{i}^{k}\right) \mathbf{e}_{\mathbf{i}}=\mathbf{0} .
$$

By (16), this implies that

$$
\lim _{k \in K} P \frac{1}{\rho_{k}} \sum_{x_{i}^{*}<0} \mu_{i}^{k} \operatorname{axp}^{\prime}\left(-\rho_{k} x_{i}^{k}\right) \mathbf{e}_{\mathbf{i}}=\mathbf{0}
$$

But, if $x_{i}^{*}<0$, then for $k$ large enough we have that $\operatorname{axp}^{\prime}\left(-\rho_{k} x_{i}^{k}\right)=-\rho_{k} x_{i}^{k}+1$. So,

$$
\lim _{k \in K} P \frac{1}{\rho_{k}} \sum_{x_{i}^{*}<0} \mu_{i}^{k}\left(-\rho_{k} x_{i}^{k}+1\right) \mathbf{e}_{\mathbf{i}}=\mathbf{0}
$$


Thus,

$$
\lim _{k \in K} P \sum_{x_{i}^{*}<0} \mu_{i}^{k}\left(-x_{i}^{k}+\frac{1}{\rho_{k}}\right) \mathbf{e}_{\mathbf{i}}=\mathbf{0} .
$$

Therefore, since $\mu_{i}^{k}$ is bounded above,

$$
\lim _{k \in K} P \sum_{x_{i}^{*}<0} \mu_{i}^{k}\left(-x_{i}^{k}\right) \mathbf{e}_{\mathbf{i}}=\mathbf{0} .
$$

Let $K_{1}$ be an infinite subset of $K$ such that

$$
\lim _{k \in K_{1}} \mu_{k}=\mu^{*}>0
$$

Taking limits for $k \in K_{1}$ in (17), we obtain:

$$
\lim _{k \in K} P \sum_{x_{i}^{*}<0} \mu_{i}^{*}\left(-x_{i}^{*}\right) \mathbf{e}_{\mathbf{i}}=\mathbf{0} .
$$

This implies the thesis.

Corollary 1. Assume that the feasible set $\left\{x \in \mathbb{R}^{n} \mid A x=b, x \geq 0\right\}$ is nonempty, $\left\{x^{k}\right\}_{k \in \mathbb{N}}$ is generated by Algorithm 4 and $x^{*}$ is a limit point. Then, $x^{*}$ is feasible.

Proof. By Theorem 2, $x^{*}$ is a stationary point of

$$
\text { Minimize } \sum_{i=1}^{n} \max \left\{0,-\mu_{i}^{*} x_{i}\right\}^{2} \text { s.t. } A x=b
$$

with $\mu^{*}>0$. But, since this problem is convex and the (nonnegative) objective function vanishes at some (feasible) point, it must vanish at every stationary point. This completes the proof.

Lemma 2. Assume that the feasible set $\left\{x \in \mathbb{R}^{n} \mid A x=b, x \geq 0\right\}$ is nonempty, $\left\{x^{k}\right\}_{k \in \mathbb{N}}$ is generated by Algorithm 4 and $K$ is an infinite set of indices such that

$$
\lim _{k \in K} x^{k}=x^{*} .
$$

Then, for all $i=1, \ldots, n$,

$$
\lim _{k \in K} \min \left\{x_{i}^{k}, \underline{\mu}_{i}^{k}\right\}=0 .
$$

Proof. If $\left\{\rho_{k}\right\}_{k \in \mathbb{N}}$ is bounded, the thesis follows from Step 5 of Algorithm 4.

Consider the case in which $\rho_{k} \rightarrow \infty$.

By Corollary $1, x^{*}$ is feasible, so $x_{i}^{*} \geq 0$ for all $i=1, \ldots, n$.

If $x_{i}^{*}=0$, since $\underline{\mu}_{i}^{k} \geq 0 \forall i, k$, the result is trivial. Suppose, now, that $x_{i}^{*}>0$. Then, taking $k \in K$ large enough,

$$
x_{i}^{k} \geq \frac{x_{i}^{*}}{2}>0 .
$$


So,

$$
\lim _{k \in K} \rho_{k} x_{i}^{k}=\infty
$$

Then,

$$
\lim _{k \in K} \operatorname{axp}^{\prime}\left(-\rho_{k} x_{i}^{k}\right)=0 .
$$

Since $\mu_{i}^{k} \leq \mu_{\max } \forall i, k$ this implies that

$$
\lim _{k \in K} \underline{\mu}_{i}^{k}=0
$$

This implies the desired result.

Theorem 3. Assume that

$$
\left\{x \in \mathbb{R}^{n} \mid A x=b, x>0\right\} \neq \emptyset,
$$

the sequence $\left\{x^{k}\right\}_{k \in \mathbb{N}}$ is generated by Algorithm 4 and $K$ is an infinite set of indices such that

$$
\lim _{k \in K} x^{k}=x^{*} .
$$

Then, $x^{*}$ is a stationary point of (11).

Proof. By Corollary 1, $A x^{*}=b, x^{*} \geq 0$ and, by Lemma 2,

$$
\lim _{k \in K} \min \left\{x_{i}^{k}, \underline{\mu}_{i}^{k}\right\}=0
$$

for all $i=1, \ldots, n$.

Moreover, by the definition of Algorithm 4,

$$
\underline{\mu}_{i}^{k} \geq 0 \forall i=1, \ldots, n, k \in \mathbb{N}
$$

and

$$
A x^{k}=b \forall k \in \mathbb{N} .
$$

By Lemma 1 and the definition of $\underline{\mu}^{k}$,

$$
\lim _{k \in K} P\left[\nabla f\left(x^{k}\right)-\sum_{i=1}^{n} \underline{\mu}_{i}^{k} \mathbf{e}_{\mathbf{i}}\right]=\mathbf{0} .
$$

So,

$$
P\left[\nabla f\left(x_{k}\right)-\underline{\mu}^{k}\right] \equiv \varepsilon_{k}^{\prime} \rightarrow 0 \text { for } k \in K .
$$

But

$$
P\left[\nabla f\left(x_{k}\right)-\underline{\mu}^{k}\right]=\nabla f\left(x^{k}\right)-\underline{\mu}^{k}-A^{T} \lambda^{k}
$$

for some $\lambda^{k} \in \mathbb{R}^{m}$, therefore,

$$
\nabla f\left(x_{k}\right)-A^{T} \lambda^{k}-\underline{\mu}^{k} \equiv \varepsilon_{k}^{\prime} \rightarrow 0 \text { for } k \in K .
$$


Therefore, for all $k \in K$, we have:

$$
\begin{gathered}
\nabla f\left(x^{k}\right)-\varepsilon_{k}^{\prime}-A^{T} \lambda^{k}-\underline{\mu}^{k}=0, \\
A x^{k}=b, \\
\underline{\mu}^{k} \geq 0,
\end{gathered}
$$

where

$$
\lim _{k \in K} \varepsilon_{k}^{\prime}=0
$$

and

$$
\lim _{k \in K} \min \left\{x_{i}^{k}, \underline{\mu}_{i}^{k}\right\}=0 \quad \forall i=1, \ldots, n .
$$

Now, for all $k \in K$ we have that $x_{1}^{k} \geq \underline{\mu}_{1}^{k}$ or $x_{1}^{k}<\underline{\mu}_{1}^{k}$, therefore, one of the following two possibilities hold:

(i) There exists an infinite set of indices $K_{1} \subset K$ such that $x_{1}^{k} \geq \underline{\mu}_{1}^{k}$ for all $k \in K_{1}$;

(ii) There exists an infinite set of indices $K_{1} \subset K$ such that $x_{1}^{k}<\underline{\mu}_{1}^{k}$ for all $k \in K_{1}$.

Using the same argument, we see that one of the following two possibilities hold:

(iii) There exists an infinite set of indices $K_{2} \subset K_{1}$ such that $x_{2}^{k} \geq \mu_{2}^{k}$ for all $k \in K_{2}$;

(iv) There exists an infinite set of indices $K_{2} \subset K_{1}$ such that $x_{2}^{k}<\underline{\mu}_{2}^{k}$ for all $k \in K_{2}$.

Continuing this extraction of subsequences for $i=1, \ldots, n$ we finally obtain an infinite set of indices $K_{n} \subset K$ such that for all $i=1, \ldots, n$ one of the following two possibilities hold:

$$
\begin{gathered}
x_{i}^{k} \geq \underline{\mu}_{i}^{k} \geq 0 \forall k \in K_{n} \\
\text { or } \\
x_{i}^{k}<\underline{\mu}_{i}^{k} \forall k \in K_{n} .
\end{gathered}
$$

So, given $i \in\{1, \ldots, n\}$, if (24) holds we have that, for all $k \in K_{n}$,

$$
\begin{gathered}
{\left[\nabla f\left(x^{k}\right)\right]_{i}-\left[\varepsilon_{k}^{\prime}+\underline{\mu}^{k}\right]_{i}-\left[A^{T} \lambda^{k}\right]_{i}=0,} \\
x_{i}^{k} \geq 0 \\
A x^{k}=b
\end{gathered}
$$

where, by (23),

$$
\lim _{k \in K_{n}}\left[\varepsilon_{k}^{\prime}+\underline{\mu}^{k}\right]_{i}=0
$$


On the other hand, if (25) holds, then for all $k \in K_{n}$,

$$
\begin{gathered}
{\left[\nabla f\left(x^{k}\right)-\varepsilon_{k}^{\prime}\right]_{i}-\left[A^{T} \lambda^{k}\right]_{i}-\underline{\mu}_{i}^{k}=0,} \\
\underline{\mu}_{i}^{k} \geq 0,
\end{gathered}
$$

and

$$
A x^{k}=b,
$$

where

$$
\lim _{k \in K_{n}} \varepsilon_{k}^{\prime}=0
$$

and

$$
\lim _{k \in K_{n}} x_{i}^{k}=0
$$

Define

$$
\begin{gathered}
I_{1}=\left\{i \in\{1, \ldots, n\} \mid x_{i}^{k} \geq \underline{\mu}_{i}^{k} \forall k \in K_{n}\right\}, \\
I_{2}=\left\{i \in\{1, \ldots, n\} \mid x_{i}^{k}<\underline{\mu}_{i}^{k} \forall k \in K_{n}\right\}, \\
{\left[\varepsilon_{k}^{\prime \prime}\right]_{i}=-\left[\varepsilon_{k}^{\prime}+\underline{\mu}^{k}\right]_{i} \text { if } i \in I_{1}}
\end{gathered}
$$

and

$$
\left[\varepsilon_{k}^{\prime \prime}\right]_{i}=-\left[\varepsilon_{k}^{\prime}\right]_{i} \text { if } i \in I_{2} .
$$

By (26)-(34), for all $k \in K_{n}, x^{k}$ is a solution of the linear programming problem:

$$
\text { Minimize }\left\langle\nabla f\left(x^{k}\right)+\varepsilon_{k}^{\prime \prime}, x-x^{k}\right\rangle
$$

subject to

$$
\begin{gathered}
A x=b, \\
x_{i} \geq 0 \text { if } i \in I_{1}, x_{i} \geq x_{i}^{k} \text { if } i \in I_{2},
\end{gathered}
$$

where

$$
\lim _{k \in K_{n}} \varepsilon_{k}^{\prime \prime}=0
$$

and

$$
\lim _{k \in K_{n}} x_{i}^{k}=0 \forall i \in I_{2} .
$$

Now, let $z \in \mathbb{R}^{n}$ be such that $A z=b$ and $z>0$. By (39), for $k \in K_{n}$ large enough, $z$ is a feasible point of the linear programming problem (35)-(37). Therefore, since $x^{k}$ is a solution of this linear programming problem,

$$
\left\langle\nabla f\left(x^{k}\right)+\varepsilon_{k}^{\prime \prime}, z-x^{k}\right\rangle \geq 0 .
$$

By (38), taking limits on both sides of this inequality for $k \in K_{n}$, we obtain that

$$
\left\langle\nabla f\left(x^{*}\right), z-x^{*}\right\rangle \geq 0 .
$$


So, we proved that (40) holds for all $z \in \mathbb{R}^{n}$ such that $A z=b$ and $z>0$.

Let now $x \in \mathbb{R}^{n}$ be such that $A x=b, x \geq 0$. Let $w \in \mathbb{R}^{n}$ be such that $w>0$ and $A w=b$. Then

$$
A[t w+(1-t) x]=b \text { and } t w+(1-t) x>0 \forall t \in(0,1] .
$$

Therefore, by (40),

$$
\left\langle\nabla f\left(x^{*}\right), t w+(1-t) x-x^{*}\right\rangle \geq 0 \forall t \in(0,1] .
$$

Taking limits in the above inequality for $t \rightarrow 0$, we obtain:

$$
\left\langle\nabla f\left(x^{*}\right), x-x^{*}\right\rangle \geq 0 .
$$

Since $x$ was an arbitrary feasible point, this implies the desired result.

\section{$5 \quad$ Implementation features}

This section deals with some specific algorithmic choices. We present the results of computer experiments in the next section. Let us first recall that $\mu_{i}^{k} \equiv 1$ in our implementation of outer iterations.

Computation of $\alpha_{k}$ and $d_{k}$ in Algorithm 1. The expression for $\alpha_{k}$ can be simplified when $k \geq 1$ as follows. Notice first that

$$
s_{k}^{T} B_{0} s_{k}=\frac{s_{k}^{T} B_{k} s_{k}}{\alpha_{k-1}}=\frac{\lambda_{k} s_{k}^{T} B_{k} d_{k}}{\alpha_{k-1}} .
$$

From (4) we obtain that $B_{k} d_{k}=-g_{k}-A^{T} \psi_{k}$. Since $s_{k}$ is a feasible direction, then $s_{k}^{T} A^{T}=0$, and so $s_{k}^{T} B_{k} d_{k}=-s_{k}^{T} g_{k}$. Hence,

$$
s_{k}^{T} B_{0} s_{k}=-\frac{\lambda_{k} s_{k}^{T} g_{k}}{\alpha_{k-1}}=-\frac{\lambda_{k}^{2}}{\alpha_{k-1}} d_{k}^{T} g_{k} .
$$

So, the expression for $\alpha_{k}$ can be reduced to

$$
\alpha_{k}=-\frac{d_{k}^{T} y_{k}}{\lambda_{k} d_{k}^{T} g_{k}} \alpha_{k-1} .
$$

For this new expression the vector $s_{k}$ is not required, and the calculation of $d_{k}^{T} g_{k}$ can be reused in (6) for each trial step during the nonmonotone line search.

On the other hand, the direction $d_{k}$ could be obtained by solving the system (4). If the order of the system were small it could be solved using the LU factorization with partial pivoting or the QR factorization. Using these linear solvers, the cost of solving the system would be $O\left(n^{3}\right)$ floating point operations. For large-scale linear systems this cost becomes 
prohibitive. So, it is convenient to compute $d_{k}$ by solving instead the system (10). In this case, we will solve several systems for consecutive iterations, with different independent vectors, using only one factorization. Moreover, since we want to exploit the fact that the KKT matrix is symmetric we use the subroutine MA27 of Duff and Reid [10]. This routine is suitable for solving specifically sparse symmetric indefinite linear systems. Finally, we note that the block $B_{0}$ changes when a re-initialization is needed in Step 6 of Algorithm 3 , so the KKT-matrix changes too. However the sparsity structure is constant during the whole process and we can also exploit this fact using suitable routines from MA27.

Initial point. To start Algorithm 3 we need an initial point $x_{0}$, which must satisfy the linear equality constraints $A x=b$. That point can be easily obtained solving the linear system

$$
\left[\begin{array}{cc}
-I & A^{T} \\
A & 0
\end{array}\right]\left[\begin{array}{c}
x_{0} \\
y
\end{array}\right]=\left[\begin{array}{l}
0 \\
b
\end{array}\right] .
$$

Observe that the matrix of this system has the same structure as the KKT matrix of (4). So, we used again the subroutine MA27.

Stopping criterion. The outer exponential algorithm was stopped when, at a solution of (12), we have that

$$
x_{i} \geq-\varepsilon \forall i=1, \ldots, n
$$

and, for all $i=1, \ldots, n$,

$$
\operatorname{axp}^{\prime}\left(-\rho x_{i}\right) \leq \varepsilon \text { whenever } x_{i} \geq \varepsilon .
$$

Condition (41) indicates that the current outer iterate is (almost) feasible. The quantities $\operatorname{axp}^{\prime}\left(-\rho x_{i}\right)$ are estimates of the Lagrange multipliers corresponding to the constraints $-x_{i} \leq 0$. Therefore, (42) indicates that the current point satisfies (approximately) the complementarity conditions. The fact that the gradient of the objective function is (approximately) a linear combination of the gradients of the active constraints must be guaranteed by the convergence criterion used in the inner algorithm. By (8) this property will hold if $d_{k}=0$. Therefore, as inner convergence criterion of Algorithm 1, we used:

$$
\left\|d_{k}\right\| \leq \varepsilon^{\prime} .
$$

Angle criterion. Since we do not have direct access to positive-definiteness of the matrices $Z^{T} B_{k} Z$, we must control the fact that descent directions are effectively generated in an indirect way. Here we decided to use

$$
\nabla F\left(x_{k}\right)^{T} d_{k} \leq-\theta\left\|d_{k}\right\|^{2} .
$$

Inequality (44) says that the appropriate Rayleigh quotient (see the proof of Theorem 1) related to $Z^{T} B_{k} Z$ is positive.

When (44) is not satisfied we have to consider two cases: 
1. If it happens immediately after a re-initialization $(\mathrm{k}=0)$ we define $B_{k}=B_{k}+$ $\rho I, \rho>0$ and a new factorization of the KKT-matrix is performed. This procedure is repeated until the angle condition is satisfied.

2. Otherwise, we perform a re-initialization and continue with Step 2 of Algorithm 3.

Re-initialization criterion at Step 4 in Algorithm 3. Among the many possible re-starting procedures, we decided to use, after preliminary experiments, the following one, which depends of a positive integer parameter $p$ :

(a) If we are at the first outer iteration or if at the previous outer iteration the inner algorithm converged within limit iterations, then at the current outer iteration the algorithm is restarted every $p^{k}$ inner iterations.

(b) If, at the previous outer iteration, the inner algorithm did not converge after limit iterations, at the current outer iteration the algorithm is restarted every $p$ iterations.

(c) The inner algorithm is restarted if condition (44) is not satisfied.

The rationale behind (a) is that, at the first iterations of the inner algorithm, large steps are expected so that the Hessian changes more abruptly than at the final iterations. Therefore, restarts must be more frequent at the first iterations than at the final ones. However, we turn to criterion (b), that restarts the algorithm after equally spaced iterations if criterion (a) did not work well.

Several strategies can be proposed for choosing $B_{0}$ when re-initialization is recommended. Since in all our experiments $f \in C^{2}\left(\mathbb{R}^{n}\right)$ we adopt the most obvious one, that is

$$
B_{0}=\nabla^{2} F\left(x_{\text {kount }}\right) \text {. }
$$

Nonmonotone line-search. When (6) does not hold in Algorithm 2, we compute the new step size using quadratic interpolation with safeguards.

\section{$6 \quad$ Numerical experiments}

We tested our algorithm using the following family of problems. Given a positive integer $\kappa$, we define

$$
\begin{gathered}
\text { Minimize } \frac{1}{2} \sum_{i=1}^{\kappa-2}\left(x_{\kappa+i+1}-x_{\kappa+i}\right)^{2} \\
\text { subject to } x_{\kappa+i}-x_{i+1}+x_{i}=0, \quad i=1, \ldots, \kappa-1, \\
\alpha_{i} \leq x_{i} \leq \alpha_{i+1}, \quad i=1, \ldots, \kappa, \\
0.4\left(\alpha_{i+2}-\alpha_{i}\right) \leq x_{\kappa+i} \leq 0.6\left(\left(\alpha_{i+2}-\alpha_{i}\right)\right), \quad i=1, \ldots, \kappa-1,
\end{gathered}
$$

where the constants $\alpha_{i}$ are defined by $\alpha_{i}=1.0+(1.01)^{i-1}$.

These problems arise in the optimal placement of nodes in a scheme for solving ordinary differential equations with given boundary values [14]. We solve these problems for different values of $\kappa$. Notice that, for this family, the matrix $A$ has $m=\kappa-1$ rows and $n=2 \kappa-1$ columns (variables). 
For the numerical experiments we set the following default parameters:

limit $=300$, maximum number of inner iterations.

$\varepsilon=10^{-8}$, tolerance for stopping criterion for outer iterations.

$\varepsilon^{\prime}=10^{-6}$, tolerance for stopping criterion for internal iterations.

$\gamma=10^{-4}$, tolerance for nonmonotone line search.

$\theta=10^{-9}$, constant in the angle criterion.

$\alpha_{\min }=10^{-3}, \alpha_{\max }=10^{3}$, the safeguards interval for the spectral parameter $\alpha$.

We use $\rho_{0}=10$ and $\eta=10$ in Algorithm 4 .

We tried to solve all the problems using $M=0,5$ and 10 for the nonmonotone line search procedure (Algorithm 2). Note that for $M=0$ we are forcing monotone decrease of the objective function at every iteration. We consider $p=1,2,3$. The case $p=1$ means that we re-start Algorithm 1 at every iteration. Therefore, this case corresponds to Newton's method.

All the numerical experiments were run on a SUN Ultra 1 Creator in double precision FORTRAN, with the optimization option -O.

The numerical results are shown in Tables 1, 2 and 3, corresponding to different values of the parameter $p$. For each table we report the parameter $\kappa$, the outer iterations (Outer), the inner iterations (Inner) and the CPU time in seconds (Time) for $M=0,5,10$. Table 4 shows the performance of an implementation of the Stationary-Newton Method, with $M=10$ and $p=2,3$. We report the number of outer iterations, inner iterations and the CPU time for the same set of problems.

Finally, in Table 5 we compare the CPU time obtained with our method ( $p=2,3$, and $M=0,10$.) against the well-known package LANCELOT $[8,9]$. We used the following parameters for LANCELOT:

- exact-second-derivatives-used

- cg-method-used

- inexact-Cauchy-point-required

- infinity-norm-trust-region-used

- gradient-tolerance 1.0D-06

- constraints-tolerance 1.0D-06

In all the experiments we verify that the three methods that we used (spectral gradient, stationary Newton and LANCELOT) converge to the same solution.

Now, we proceed to the analysis of these tables. First of all, we consider Tables 1-3 separately. Table $1(p=1)$ shows that there is no difference between monotone $(M=0)$ and nonmonotone versions $(M=5,10)$, except for problems with $\kappa=650$ and $\kappa=800$. Clearly, $p=1, M=0$ corresponds, essentially, to a projected Newton's method with backtracking (and modified exponential penalization). On the other hand, Tables 2 and 3 ( $p=2$ and 3 respectively) show that the nonmonotone version was better than the monotone one, specially for large scale problems. 


\begin{tabular}{|r|r|r|r|r|r|r|r|r|r|}
\hline & \multicolumn{3}{|c|}{$\mathrm{M}=0$} & \multicolumn{3}{|c|}{$\mathrm{M}=5$} & \multicolumn{3}{|c|}{$\mathrm{M}=10$} \\
\hline$\kappa$ & Outer & Inner & Time & Outer & Inner & Time & Outer & Inner & Time \\
\hline \hline 50 & 5 & 38 & 0.14 & 5 & 32 & 0.11 & 5 & 32 & 0.11 \\
100 & 5 & 42 & 0.23 & 5 & 55 & 0.26 & 5 & 57 & 0.27 \\
150 & 5 & 48 & 0.38 & 5 & 62 & 0.43 & 5 & 65 & 0.45 \\
200 & 5 & 80 & 0.80 & 5 & 67 & 0.65 & 5 & 67 & 0.65 \\
250 & 5 & 81 & 1.08 & 5 & 84 & 1.11 & 5 & 84 & 1.11 \\
300 & 5 & 90 & 1.47 & 5 & 96 & 1.50 & 5 & 95 & 1.49 \\
350 & 5 & 77 & 1.53 & 5 & 100 & 1.85 & 5 & 113 & 2.04 \\
400 & 5 & 148 & 3.35 & 5 & 120 & 2.55 & 5 & 120 & 2.55 \\
450 & 5 & 136 & 3.38 & 5 & 133 & 3.29 & 5 & 138 & 3.38 \\
500 & 5 & 139 & 4.05 & 5 & 139 & 4.01 & 5 & 141 & 4.00 \\
550 & 5 & 320 & 12.56 & 5 & 711 & 31.39 & 5 & 688 & 30.60 \\
600 & 5 & 704 & 34.44 & 5 & 732 & 34.94 & 5 & 718 & 34.46 \\
650 & 5 & 623 & 33.16 & 5 & 703 & 38.10 & 5 & 549 & 28.79 \\
700 & 5 & 722 & 59.28 & 5 & 719 & 58.84 & 5 & 715 & 58.65 \\
750 & 5 & 739 & 45.88 & 5 & 739 & 45.46 & 5 & 732 & 45.28 \\
800 & 5 & 731 & 48.62 & 5 & 754 & 48.82 & 5 & 609 & 37.92 \\
850 & 4 & 449 & 42.52 & 4 & 441 & 41.67 & 4 & 444 & 41.80 \\
900 & 4 & 441 & 32.73 & 4 & 439 & 32.40 & 4 & 439 & 32.43 \\
\hline
\end{tabular}

Table 1: Spectral Method, $p=1$ (NEWTON)

\begin{tabular}{|r|r|r|r|r|r|r|r|r|r|}
\hline & \multicolumn{3}{|c|}{$\mathrm{M}=0$} & \multicolumn{3}{|c|}{$\mathrm{M}=5$} & \multicolumn{3}{|c|}{$\mathrm{M}=10$} \\
\hline$\kappa$ & Outer & Inner & Time & Outer & Inner & Time & Outer & Inner & Time \\
\hline \hline 50 & 5 & 204 & 0.20 & 5 & 65 & 0.09 & 5 & 62 & 0.09 \\
100 & 5 & 148 & 0.32 & 5 & 127 & 0.24 & 5 & 136 & 0.25 \\
150 & 5 & 147 & 0.45 & 5 & 266 & 0.67 & 5 & 225 & 0.57 \\
200 & 5 & 342 & 1.30 & 5 & 368 & 1.46 & 5 & 366 & 1.44 \\
250 & 5 & 350 & 1.77 & 5 & 361 & 1.77 & 5 & 353 & 1.75 \\
300 & 5 & 380 & 2.44 & 5 & 396 & 2.49 & 5 & 425 & 2.90 \\
350 & 5 & 527 & 4.34 & 5 & 487 & 4.17 & 5 & 563 & 4.93 \\
400 & 5 & 404 & 3.92 & 5 & 415 & 4.03 & 5 & 493 & 5.69 \\
450 & 4 & 605 & 7.10 & 4 & 570 & 6.82 & 4 & 630 & 7.67 \\
500 & 5 & 447 & 5.83 & 5 & 458 & 5.97 & 5 & 486 & 6.55 \\
550 & 5 & 860 & 16.34 & 5 & 641 & 9.00 & 5 & 696 & 9.30 \\
600 & 5 & 764 & 11.58 & 5 & 715 & 11.40 & 5 & 696 & 10.96 \\
650 & 5 & 934 & 15.16 & 5 & 796 & 14.57 & 5 & 915 & 16.77 \\
700 & 5 & 974 & 25.96 & 5 & 956 & 22.46 & 5 & 1270 & 47.13 \\
750 & 6 & 888 & 20.15 & 5 & 901 & 18.14 & 5 & 880 & 18.84 \\
800 & 5 & 975 & 25.00 & 5 & 1303 & 44.40 & 5 & 909 & 20.98 \\
850 & 5 & 1346 & 54.73 & 5 & 916 & 25.06 & 4 & 899 & 23.18 \\
900 & 5 & 973 & 24.74 & 5 & 1392 & 54.48 & 5 & 989 & 26.68 \\
\hline
\end{tabular}

Table 2: Spectral Method, $p=2$ 


\begin{tabular}{|r|r|r|r|r|r|r|r|r|r|}
\hline & \multicolumn{3}{|c|}{$\mathrm{M}=0$} & \multicolumn{3}{|c|}{$\mathrm{M}=5$} & \multicolumn{3}{|c|}{$\mathrm{M}=10$} \\
\hline$\kappa$ & Outer & Inner & Time & Outer & Inner & Time & Outer & Inner & Time \\
\hline \hline 50 & 5 & 318 & 0.30 & 5 & 144 & 0.15 & 5 & 222 & 0.20 \\
100 & 5 & 183 & 0.36 & 5 & 295 & 0.50 & 5 & 330 & 0.55 \\
150 & 5 & 266 & 0.73 & 5 & 282 & 0.69 & 5 & 323 & 0.80 \\
200 & 5 & 371 & 1.34 & 5 & 360 & 1.33 & 5 & 407 & 1.70 \\
250 & 5 & 342 & 1.67 & 5 & 434 & 2.18 & 5 & 434 & 2.15 \\
300 & 5 & 488 & 3.59 & 5 & 560 & 3.33 & 4 & 477 & 2.98 \\
350 & 5 & 606 & 4.92 & 5 & 457 & 3.64 & 5 & 465 & 3.70 \\
400 & 5 & 444 & 4.24 & 5 & 452 & 4.29 & 5 & 485 & 4.66 \\
450 & 4 & 624 & 6.91 & 5 & 534 & 5.72 & 5 & 543 & 5.72 \\
500 & 5 & 525 & 6.62 & 5 & 546 & 7.08 & 5 & 531 & 6.93 \\
550 & 5 & 954 & 14.97 & 5 & 753 & 10.23 & 5 & 716 & 9.73 \\
600 & 5 & 907 & 12.75 & 5 & 745 & 11.44 & 5 & 827 & 12.31 \\
650 & 5 & 965 & 15.45 & 5 & 888 & 15.53 & 5 & 907 & 14.94 \\
700 & 5 & 1030 & 24.89 & 6 & 914 & 14.90 & 6 & 908 & 17.98 \\
750 & 5 & 967 & 17.70 & 5 & 842 & 17.00 & 6 & 905 & 19.25 \\
800 & 5 & 1231 & 27.56 & 6 & 1046 & 22.22 & 6 & 1039 & 22.25 \\
850 & 5 & 1125 & 31.38 & 4 & 977 & 23.10 & 5 & 930 & 24.53 \\
900 & 5 & 858 & 23.54 & 5 & 956 & 21.92 & 5 & 1026 & 25.05 \\
\hline
\end{tabular}

Table 3: Spectral Method, $p=3$

\begin{tabular}{|r|r|r|r|r|r|r|}
\hline & \multicolumn{3}{|c|}{$p=2$} & \multicolumn{3}{|c|}{$p=3$} \\
\hline$\kappa$ & Outer & Inner & Time & Outer & Inner & Time \\
\hline \hline 50 & 5 & 264 & 0.19 & 5 & 309 & 0.19 \\
100 & 5 & 310 & 0.47 & 5 & 322 & 0.40 \\
150 & 5 & 327 & 0.78 & 5 & 328 & 0.63 \\
200 & 5 & 410 & 1.52 & 4 & 463 & 1.48 \\
250 & 5 & 568 & 2.38 & 5 & 386 & 1.57 \\
300 & 5 & 648 & 3.93 & 5 & 412 & 2.16 \\
350 & 5 & 585 & 4.50 & 5 & 411 & 2.57 \\
400 & 5 & 877 & 10.26 & 5 & 479 & 4.16 \\
450 & 5 & 520 & 4.85 & 5 & 686 & 5.92 \\
500 & 5 & 697 & 7.72 & 5 & 721 & 8.17 \\
550 & 5 & 1067 & 23.24 & 5 & 1074 & 14.37 \\
600 & 5 & 1115 & 19.51 & 5 & 1163 & 17.32 \\
650 & 5 & 1275 & 31.66 & 5 & 939 & 16.17 \\
700 & 5 & 1169 & 24.89 & 5 & 1236 & 21.17 \\
750 & 5 & 1462 & 40.16 & 5 & 1298 & 33.54 \\
800 & 5 & 1376 & 40.07 & 5 & 875 & 20.93 \\
850 & 5 & 1322 & 45.85 & 5 & 1287 & 38.05 \\
900 & 5 & 1077 & 33.15 & 5 & 1288 & 40.76 \\
\hline
\end{tabular}

Table 4: Stationary-Newton, $M=10$ 


\begin{tabular}{|r|r|r|r|r|r|}
\hline & \multicolumn{2}{|c|}{$\mathrm{p}=2$} & \multicolumn{2}{c|}{$\mathrm{p}=3$} & \multirow{2}{*}{ LANCELOT } \\
\hline$\kappa$ & $M=0$ & $M=10$ & $M=0$ & $M=10$ & \\
\hline \hline 50 & 0.20 & 0.09 & 0.30 & 0.20 & 1.07 \\
100 & 0.32 & 0.25 & 0.36 & 0.55 & 5.98 \\
150 & 0.45 & 0.57 & 0.73 & 0.80 & 14.63 \\
200 & 1.30 & 1.44 & 1.34 & 1.70 & 33.64 \\
250 & 1.77 & 1.75 & 1.67 & 2.15 & 36.69 \\
300 & 2.44 & 2.90 & 3.59 & 2.98 & 52.81 \\
350 & 4.34 & 4.93 & 4.92 & 3.70 & 68.03 \\
400 & 3.92 & 5.69 & 4.24 & 4.66 & 115.01 \\
450 & 7.10 & 7.67 & 6.91 & 5.72 & 93.12 \\
500 & 5.83 & 6.55 & 6.62 & 6.93 & 167.43 \\
550 & 16.34 & 9.30 & 14.97 & 9.73 & 210.72 \\
600 & 11.58 & 10.96 & 12.75 & 12.31 & 242.94 \\
650 & 15.16 & 16.77 & 15.45 & 14.94 & 263.84 \\
700 & 25.96 & 47.13 & 24.89 & 17.98 & 331.65 \\
750 & 20.15 & 18.84 & 17.70 & 19.25 & 349.01 \\
800 & 25.00 & 20.98 & 27.56 & 22.25 & 351.11 \\
850 & 54.73 & 23.18 & 31.38 & 24.53 & 430.93 \\
900 & 24.74 & 26.68 & 23.54 & 25.05 & 475.93 \\
\hline
\end{tabular}

Table 5: CPU time of Spectral Method vs. CPU time of LANCELOT

Now, if we consider Tables 1-3 jointly we note that in most of the experiments the number of inner iterations for $p=2$ and 3 was greater than for $p=1$. However, for large scale problems, the CPU time for $p=2$ and 3 and nonmonotone versions were less than those corresponding to the case $p=1$.

Comparing the previous tables with Table 4 shows that performing the spectral correction of the Hessian at inner iterations is better than not correcting at all, as the Stationary Newton method does.

Finally, Table 5 shows that using any implementation of the spectral method ( $p=2$ or 3 ) in the monotone or nonmonotone line search is more effective than using LANCELOT (with the mentioned parameters) on this set of problems.

Let us try to explain the reasons for the observed results.

(1) The performance of our method was better than Newton's method except for small problems. (Observe that the sparsity pattern of the matrix (4) is suitable for a rather quick factorization using MA27.) The fact that Newton's method, in any of its variations, is very effective when the linear algebra associated to it is not prohibitive is well known in numerical optimization. Moreover, in that case, the association of Newton with the logarithmic barrier function (see, for example $[23,24]$ ) is usually profitable. It would be easy to construct examples where the situation is completely different, just placing an objective function with a dense or badly structured Hessian. On the other hand, the fact that in our method we do not factorize the KKT-matrix at each iteration is a very important advantage, as can be clearly observed comparing the CPU time for large scale problems. 
(2) More important is the fact that the spectral preconditioned methods outperformed stationary Newton variations. In fact, stationary Newton methods (with restarts) are good alternatives to Newton when this is not affordable. So, it is interesting to observe that with minimal corrections, which do not increase the computational cost of Newton's stationary methods, we can obtain significant improvements of the overall performance.

(3) The fact that explains the superiority of the methods introduced in this paper against LANCELOT is, essentially, that the matrix $A$ has a structure that makes the factorization of (4) affordable. LANCELOT does not use factorizations at all and, so, this advantage is lost.

(4) As in $[6,15,22]$ the numerical results for large scale problems using a nonmonotone line search were better than those using monotone ones.

\section{$7 \quad$ Final remarks}

As we mentioned in the introduction, spectral gradient methods for unconstrained and bound constrained problems have shown to be very effective when compared to conjugate gradient methods and with other methods especially designed for large scale problems. See $[6,22]$. In fact, as we can see in Tables $1-5$ the performance of our method was satisfactory for small scale problems and very attractive and encouraging for large scale problems.

Given the observations and possible explanations in section 6, let us forecast the practical future of algorithms like the ones presented in this paper. First of all, it is clear to us that spectral gradient algorithms are not going to replace Newton's method, when the linear algebra cost associated to the latter is moderate. However, in situations where a Newton iteration can be performed but is rather expensive, a spectral Hessian scaling performed at most iterations must be more effective than merely repeating the previous Hessian.

If a factorization of (4) is so expensive that it cannot be performed even in a single iteration, two different situations can be distinguished. In the first situation, the matrix responsible for the cost associated to the factorization of (4) is the constraint matrix $A$. In this case, Algorithm 1 cannot be implemented either. This is the case in which it is recommendable to incorporate the constraints $A x=b$ to the objective function, as LANCELOT does (see $[8,16])$. In the second case, the Hessian of the objective function is the one that makes the factorization (4) impossible. In this case, the factorization of

$$
\left[\begin{array}{cc}
I & A^{T} \\
A & 0
\end{array}\right]
$$

is probably affordable, and the spectral gradient method based on $B_{k} \equiv I$ can be comparatively efficient. In an extreme case, the problem is unconstrained $(m=0)$, a situation where the spectral gradient technique has proved to be effective.

Finally, a few words must be said with respect to the penalty strategy used here. There exist at least two completely different strategies for dealing with the bounds. The most classical one is the active set strategy described, for example, in $[11,13]$ and many other 
text books and papers. In the last decade, interior point algorithms like the ones described in [24] became increasingly popular. The association of interior point (logarithmic barrier) algorithms with Newtonian directions seems to be very effective. However, this does not seem to be the case when Newtonian directions cannot be computed at all. In this case, we feel that the modified exponential penalization is a good alternative although much research is necessary in order to balance the termination criterion of the inner algorithm with the global convergence criterion.

\section{Acknowledgements.}

We would like to thank two anonymous referees whose comments helped us to improve the final version of this paper.

\section{References}

[1] J. Barzilai and J. M. Borwein [1988], Two point step size gradient methods, IMA Journal of Numerical Analysis 8, pp. 141-148.

[2] D. P. Bertsekas [1995], Nonlinear Programming, Athena Scientific, Belmont, MA.

[3] R. H. Bielschowsky, A. Friedlander, F. A. M. Gomes, J. M. Martínez and M. Raydan [1997], An adaptive algorithm for bound constrained quadratic minimization, Investigación Operativa 7, pp. 67-102.

[4] E. G. Birgin, I. Chambouleyron and J. M. Martínez [1999], Estimation of the optical constants and the thickness of thin films using unconstrained optimization, Journal of Computational Physics 151, pp. 862-880.

[5] E. G. Birgin and Y. G. Evtushenko [1998], Automatic differentiation and spectral projected gradient methods for optimal control problems, Optimization Methods and Software 10, pp. 125-146.

[6] E. G. Birgin, J. M. Martínez and M. Raydan [2000], Nonmonotone Spectral Projected Gradient Methods on Convex Sets, SIAM Journal on Optimization 10, pp. 1196-1211.

[7] E. G. Birgin, J. M. Martínez and M. Raydan [2003]. Inexact spectral projected gradient methods on convex sets. To appear in IMA Journal of Numerical Analysis.

[8] A. R. Conn, N. I. M. Gould and Ph. L. Toint [1991], A globally convergent augmented Lagrangian algorithm for optimization with general constraints and simple bounds, SIAM Journal on Numerical Analysis 28, pp. 545 - 572.

[9] A. R. Conn, N. I. M. Gould and Ph. L. Toint [1992], LANCELOT: a Fortran package for large-scale nonlinear optimization (Release A), Springer Series in Computational Mathematics 17, Springer Verlag, Heidelberg, Berlin, New York. 
[10] I. S. Duff and J. K. Reid [1982], MA27 - a set of Fortran subroutines for solving sparse symmetric sets of linear equations, Technical Report R-10533, Computer Science and System Division, AERE Harwell, Oxford, England.

[11] R. Fletcher [1987], Practical Methods of Optimization, Wiley, New York.

[12] A. Friedlander, J. M. Martínez and M. Raydan [1995], A new method for large-scale box constrained convex quadratic minimization problems, Optimization Methods and Software 5, pp. 57-74.

[13] P. E. Gill, W. Murray and M. H. Wright [1981], Practical optimization, Academic Press, London, England.

[14] N. I. M. Gould [1991] An algorithm for large-scale quadratic programming, IMA Journal of Numerical Analysis 11, pp. 299-324.

[15] L. Grippo, F. Lampariello and S. Lucidi [1986], A nonmonotone line search technique for Newton's method, SIAM Journal on Numerical Analysis 23, pp. 707-716.

[16] N. Krejić, J. M. Martínez, M. P. Mello and E. A. Pilotta [2000], Validation of an augmented Lagrangian algorithm with a Gauss-Newton Hessian approximation using a set of hard-spheres problems, Journal of Computational Optimization and Applications 16, pp. 247-263.

[17] F. Luengo, M. Raydan, W. Glunt and T.L. Hayden [2002], Preconditioned spectral gradient method, Numerical Algorithms 30, pp. 241-258.

[18] J. M. Martínez [2000], BOX-QUACAN and the implementation of Augmented Lagrangian algorithms for minimization with inequality constraints, Computational and Applied Mathematics 19, pp. 31-56.

[19] B. Molina and M. Raydan [1996], The preconditioned Barzilai-Borwein method for the numerical solution of partial differential equations, Numerical Algorithms 13, pp. 45-60.

[20] F. H. Murphy [1974], A class of exponential penalty functions, SIAM Journal on Control 12, pp. 679-687.

[21] M. Raydan [1993], On the Barzilai and Borwein choice of steplength for the gradient method, IMA Journal of Numerical Analysis 13, pp. 321-326.

[22] M. Raydan [1997], The Barzilai and Borwein gradient method for the large scale unconstrained minimization problem, SIAM Journal on Optimization 7, pp. 26-33.

[23] R. J. Vanderbei [1994], LOQO: An interior point code for quadratic programming, Technical Report SOR-94-15, Princeton University.

[24] R. J. Vanderbei and D. F. Shanno [1997]: An interior point algorithm for nonconvex nonlinear programming, Technical Report SOR-97-21, Statistics and Operations Research, Princeton University. 